\title{
Psychometric evaluation of the Polish adaptation of the Hill-Bone Compliance to High Blood Pressure Therapy Scale
}

\author{
Izabella Uchmanowicz', Beata Jankowska-Polańska', Anna Chudiak', Anna Szymańska-Chabowska²*
} and Grzegorz Mazur ${ }^{2}$

\begin{abstract}
Background: Development of simple instruments for the determination of the level of adherence in patients with high blood pressure is the subject of ongoing research. One such instrument, gaining growing popularity worldwide, is the Hill-Bone Compliance to High Blood Pressure Therapy.

The aim of this study was to adapt and to test the reliability of the Polish version of Hill-Bone Compliance to High Blood Pressure Therapy Scale.

Methods: A standard guideline was used for the translation and cultural adaptation of the English version of the Hill-Bone Compliance to High Blood Pressure Therapy Scale into Polish. The study included 117 Polish patients with hypertension aged between 27 and 90 years, among them 53 men and 64 women. Cronbach's alpha was used for analysing the internal consistency of the scale.

Results: The mean score in the reduced sodium intake subscale was $M=5.7$ points (standard deviation SD $=1.6$ points). The mean score in the appointment-keeping subscale was $M=3.4$ points (standard deviation SD $=1.4$ points). The mean score in the medication-taking subscale was $M=11.6$ points (standard deviation SD = 3.3 points). In the principal component analysis, the three-factor system (1 - medication-taking, 2 - appointment-keeping, 3 reduced sodium intake) accounted for $53 \%$ of total variance. All questions had factor loadings $>0.4$. The medication-taking subscale: most questions (6 out of 9) had the highest loadings with Factor 1. The appointmentkeeping subscale: all questions (2 out of 2) had the highest loadings with Factor 2. The reduced sodium intake subscale: most questions (2 out of 3 ) had the highest loadings with Factor 3. Goodness of fit was tested at $c h i^{2}=248.87 ; p<0.001$. The Cronbach's alpha score for the entire questionnaire was 0.851 .
\end{abstract}

Conclusion: The Hill-Bone Compliance to High Blood Pressure Therapy Scale proved to be suitable for use in the Polish population. Use of this screening tool for the assessment of adherence to BP treatment is recommended.

Keywords: Hypertension, Adherence, Hill-Bone Compliance to High Blood Pressure Therapy Scale, Internal consistency, Reliability

\footnotetext{
* Correspondence: aszyman@mp.pl

2Department of Internal Medicine, Occupational Diseases and Hypertension,

Wroclaw Medical University, 213 Borowska Street, 50-556 Wroclaw, Poland

Full list of author information is available at the end of the article
} 


\section{Background}

Hypertension, or high blood pressure (HBP), is considered the most common disorder in the general population. The World Health Organization (WHO) Report for 2008 states that $40 \%$ of the world's population over the age of 25 have high blood pressure [1]. Worldwide, HBP affects over 1.5 billion people, in Poland - approximately 8.6 million, i.e. $29 \%$ of adults. According to estimates, in 2025, over 2 billion people will suffer from HBP [2]. Despite the wealth of knowledge and the advances in pharmaceutical treatment and hypotensive drugs, the effectiveness of HBP treatment in Poland is as low as 5$15 \%$ [3]. Non-adherence to medications is considered one of the largest related issues $[4,5]$. Several methods can be used to test compliance and adherence, including pharmacological, clinical and physical ones. The first method consists of determining the concentrations of drugs or their metabolites in serum or in urine. The clinical methods are based on monitoring follow-up appointment-keeping. It is assumed that patients who are cooperative and follow their physicians' advice tend to keep their follow-up appointments. The remaining methods used for determining adherence and compliance are physical ones, consisting in counting the tablets taken or using tablet-counting systems. These methods are not, however, reliable, as the presentation of an empty medicine package to the physician is no proof that all the doses were taken as prescribed. Patients may deliberately dispose of the medicine, which artificially increases the score [6-8]. Remote observation is an important aspect of compliance and adherence assessment among patients with HBP. After starting pharmaceutical treatment, follow-up visits should take place every 24 weeks to enable assessment of the drugs' effectiveness and any side effects. Once blood pressure falls to the desired values, these follow-ups may be planned at longer intervals, every few months [9-11]. Every 2 years, all patients should be checked for risk factors and end-organ complications of HBP [12]. Physicians'lack of knowledge and patients'lack of awareness account for about $70 \%$ of non-adherence, indicating the necessity to improve physician education and patient involvement [13, 14]. Therefore, the researchers work on simple instruments to assess compliance and adherence to treatment in this group of patients. One such instrument, increasing in popularity worldwide, is the Hill-Bone Compliance to High Blood Pressure Therapy Scale [15]. In its current version, the Hill-Bone Compliance to High Blood Pressure Therapy Scale comprises 14 questions that are grouped in the following sub-scales: reduced sodium intake (three items), appointment-keeping (two items), and medication-taking (nine items) [15]. The instrument has successfully passed validation and psychometric evaluation in many populations and cultures, and has undergone multiple translations and adaptations [16-19]. We decided to perform a cultural Polish adaptation of the Hill-Bone Compliance to High Blood Pressure Therapy Scale questionnaire and to validate it in a group of HBP patients. This paper presents the results of our validation study.

\section{Methods \\ Questionnaire \\ The Polish adaptation of the Hill-Bone Compliance to High Blood Pressure Therapy Scale (HBCS)}

The adaptation was performed using the standard methodology [20]. The Polish adaptation is based on the English-language version of the scale (Table 1) [15]. Having received the authors' approval, the questionnaire was translated into Polish by two independent translators. Then, the translations were evaluated by a panel of experts, which comprised a cardiologist, a general practitioner, two specialist cardiology nurses and a psychologist. The panel verified the phrasing and meaning of all questions, as well as the clarity and correctness of the instructions. The version selected by the panel subsequently underwent back-translation and the result was submitted for approval by the authors of the original English version. Once approved, the preliminary version was used in a pilot study on a group of 30 patients diagnosed with HBP. The pilot study resulted in the final Polish version of the Hill-Bone Compliance to High

Table 1 The Hill-Bone Compliance to HBP Therapy Scale - the original English version

\begin{tabular}{ll}
\hline No. & $\begin{array}{l}\text { Item } \quad \text { Response: } \\
\text { 1. All of the time 2. Most of the time 3. Some of the } \\
\text { time 4. Never }\end{array}$ \\
\hline 1 & How often do you forget to take your HBP medicine? \\
3 & How often do you decide NOT to take your HBP medicine? \\
4 & How often do you add salt to your food before you eat it? \\
5 & How often do you eat fast food? \\
6 & How often do you make the next appointment before you \\
7 & leave the doctor's office?* \\
8 & How often do you miss scheduled appointments? \\
9 & How often do you run out of HBP pills? \\
10 & How often do you skip your HBP medicine before you go to \\
14 & the doctor?
\end{tabular}


Blood Pressure Therapy Scale, validated in the present study.

\section{Patients}

The study was carried out at a private medical center in Wrocław, between March and September, 2015, and included 117 sequential patients diagnosed with HBP in accordance with the ESC criteria. The patients filled in the questionnaire during a regular follow-up visit. The refusal rate was $0 \%$, and the acceptance rate was $100 \%$. The only exclusion criterion was cognitive impairment and/or communication barriers interfering with questionnaire completion. The sample size was based on literature data, indicating that the minimum number of participants in a validation study should be five times the number of variables analyzed [21]. Therefore, a validation study of the Hill-Bone Compliance to High Blood Pressure Therapy Scale should include a minimum of 70 patients $(5 \times 14$ statements). It was the representative sample of hypertensive patients in Poland, so it might be generalized to other patients. All patients gave informed written consent to participate in writing, and the study protocol was approved by the Independent Bioethics Committee of the Wrocław Medical University (decision no. KB 136/2015).

\section{Statistical analysis}

The results obtained were analyzed using Statistica 10 software (StatSoft, Tulsa, USA). The normality of variable distribution was verified using the KolmogorovSmirnov test, and the statistical characteristics of variables were presented using arithmetical means \pm standard deviations (SD), median values and interquartile ranges (IQR). The statistical characteristics of discrete and qualitative variables were presented using number distributions (n). The internal consistency and the intraclass correlation coefficients of the Polish adaptation were assessed based on Cronbach's alpha.

\section{Results}

The socio-demographic and clinical characteristics of the 117 patients studied are summarized in Table 2. The sample included 53 men $(45.30 \%)$ and 64 women (54.70\%). The mean age was 60.7 years (SD 12.41). Most patients were married or living with a partner $(66.67 \%)$ and high-school educated (51.28 \%). The mean time from diagnosis was 9.18 years (SD 7.4). The number of tablets taken daily was 2.61 (SD 3.26). The detailed characteristics of the patients are shown in Table 2. The Table 3 shows descriptive statistics from the Hill-Bone Compliance to High Blood Pressure Therapy Scale in the studied group.
Table 2 Patients' socio-demographic and clinical data

\begin{tabular}{|c|c|c|}
\hline Socio-demographic and clinical data & $n$ & $\%$ of total \\
\hline \multicolumn{3}{|l|}{ Sex } \\
\hline Female & 64 & 54.70 \\
\hline Male & 53 & 45.30 \\
\hline \multicolumn{3}{|l|}{ Age } \\
\hline$M \pm S D$ & $60.77 \pm 12.41$ & \\
\hline $\operatorname{Min} \div \operatorname{Max}$ & $27 \div 90$ & \\
\hline \multicolumn{3}{|l|}{ Marital status } \\
\hline Married/living with a partner & 78 & 66.67 \\
\hline Single & 39 & 33.33 \\
\hline \multicolumn{3}{|l|}{ Education } \\
\hline None or primary & 32 & 27.35 \\
\hline High school & 60 & 51.28 \\
\hline Higher vocational/College/University & 25 & 21.37 \\
\hline Blood pressure value & Systolic & Diastolic \\
\hline$M \pm S D$ & $144.14 \pm 13.69$ & $87.85 \pm 11.36$ \\
\hline $\operatorname{Min} \div \operatorname{Max}$ & $120 \div 190$ & $60 \div 120$ \\
\hline \multicolumn{3}{|l|}{ Time from HBP diagnosis } \\
\hline $\mathrm{M} \pm \mathrm{SD}$ & $9.18 \pm 7.4$ & \\
\hline Min $\div$ Max & $1 \div 10$ & \\
\hline \multicolumn{3}{|l|}{ JNC blood pressure classification } \\
\hline Stage 1 hypertension & 63 & 53.85 \\
\hline Stage 2 hypertension & 36 & 30.77 \\
\hline Prehypertension & 18 & 15.38 \\
\hline
\end{tabular}

$n$ number, $M \pm S D$ mean \pm standard deviation, Min $\div$ Max minimum $\div$

maximum, $H B P$ high blood pressure, JNC Joint National Committee

The mean sodium intake score among the patients studied was $\mathrm{M}=5.7$ points (standard deviation $\mathrm{SD}=1.6$ points). The mean appointment-keeping score in the group was $\mathrm{M}=3.4$ points (standard deviation $\mathrm{SD}=1.4$ points). The mean medicine-taking score was $M=11.6$ points (standard deviation $\mathrm{SD}=3.3$ points).

\section{The scale's construct validity}

The principal components of the HBSC scale were analyzed. The three-factor system (variables: 1 medication-taking, 2 - appointment-keeping, 3 - reduced sodium intake) accounts for $53 \%$ of total variance. All questions have factor loadings $>0.4$. The medication-taking subscale: most questions (6 out of 9) have the highest loadings with Factor 1. The appointment-keeping subscale: all questions (2 out of 2 ) have the highest loadings with Factor 2. The reduced sodium intake subscale: most questions (2 out of 3 ) have the highest loadings with Factor 3. Goodness of fit was tested at $\mathrm{chi}^{2}=248.87 ; p<0.001$ (Table 4). The instrument shows appropriate construct validity and no questions need to be excluded. 
Table 3 Descriptive statistics from the Hill-Bone Compliance to High Blood Pressure Therapy Scale in the studied group

\begin{tabular}{|c|c|c|c|c|c|c|}
\hline \multicolumn{2}{|c|}{ Question } & \multirow{2}{*}{$\frac{M \pm S D}{2.41 \pm 0.76}$} & \multirow{2}{*}{$\begin{array}{l}\text { All the time [\%] } \\
6.84\end{array}$} & \multirow{2}{*}{$\begin{array}{l}\text { Most of the time [\%] } \\
53.85\end{array}$} & \multirow{2}{*}{$\frac{\text { Sometimes [\%] }}{29.06}$} & \multirow{2}{*}{$\frac{\text { Never [\%] }}{9.40}$} \\
\hline 3 & How often do you eat salty food? & & & & & \\
\hline 4 & How often do you add salt to your food before you eat it? & $1.93 \pm 0.85$ & 35.04 & 41.03 & 19.66 & 4.27 \\
\hline 5 & How often do you eat fast food? & $1.40 \pm 0.61$ & 63.25 & 29.06 & 3.42 & 0.85 \\
\hline \multicolumn{2}{|c|}{ Reduced sodium intake } & \multicolumn{2}{|l|}{$5.68 \pm 1.60$} & & & \\
\hline 6 & $\begin{array}{l}\text { How often do you make the next appointment before you } \\
\text { leave the doctor's office? }\end{array}$ & $2.75 \pm 1.05$ & 11.97 & 29.06 & 22.22 & 29.91 \\
\hline 7 & How often do you miss scheduled appointments? & $1.30 \pm 0.61$ & 74.36 & 19.66 & 2.56 & 1.71 \\
\hline \multicolumn{2}{|c|}{ Appointment-keeping } & \multicolumn{2}{|l|}{$3.41 \pm 1.45$} & & & \\
\hline 1 & How often do you forget to take your HBP medicine? & $1.39 \pm 0.64$ & 67.52 & 26.50 & 3.42 & 1.71 \\
\hline 2 & $\begin{array}{l}\text { Jak często decyduje się Pan/Pani nie brać leków na } \\
\text { nadciśnienie? [How often do you decide NOT to take } \\
\text { your HBP medicine?] }\end{array}$ & $1.35 \pm 0.62$ & 70.94 & 21.37 & 5.13 & 0.85 \\
\hline 8 & How often do you forget to get prescriptions filled? & $1.30 \pm 0.50$ & 70.94 & 25.64 & 1.71 & 0.00 \\
\hline 9 & How often do you run out of HBP pills? & $1.42 \pm 0.56$ & 60.68 & 34.19 & 3.42 & 0.00 \\
\hline 10 & $\begin{array}{l}\text { How often do you skip your HBP medicine before you } \\
\text { go to the doctor? }\end{array}$ & $1.25 \pm 0.43$ & 72.65 & 23.93 & 0.00 & 0.00 \\
\hline 1 & $\begin{array}{l}\text { How often do you miss taking your HBP pills when } \\
\text { you feel better? }\end{array}$ & $1.39 \pm 0.68$ & 68.38 & 26.50 & 0.85 & 3.42 \\
\hline 12 & $\begin{array}{l}\text { How often do you miss taking your HBP pills when } \\
\text { you feel sick? }\end{array}$ & $1.33 \pm 0.60$ & 71.79 & 21.37 & 4.27 & 0.85 \\
\hline 13 & How often do you take someone else's HBP pills? & $1.17 \pm 0.53$ & 86.32 & 7.69 & 1.71 & 1.71 \\
\hline 14 & $\begin{array}{l}\text { How often do you miss taking your HBP pills when } \\
\text { you are careless? }\end{array}$ & $1.25 \pm 0.53$ & 77.78 & 16.24 & 4.27 & 0.00 \\
\hline \multicolumn{2}{|c|}{ Medicine-taking } & $11.62 \pm 3.32$ & & & & \\
\hline
\end{tabular}

$H B P$ high blood pressure, $M \pm S D$ mean \pm standard deviation

\section{The scale's criterion validity}

Analysis of correlation with external criteria (systolic and diastolic blood pressure) - Spearman's rank correlation coefficients (Table 5). The instrument has unique criterion validity, which is a complement to the blood pressure measurement and not its substitute.

\section{Scale reliability}

Internal consistency was tested using Cronbach's alpha, and the intraclass correlation coefficient was determined. The Cronbach's alpha score for the whole questionnaire was 0.80 , and for the medicine-taking subscale it was 0.78. Cronbach's alpha was not calculated for the remaining subscales due to the insufficient number of questions. Coefficients of correlation with the total score ranged from 0.14 (question 3) to 0.653 (question 10). The intraclass correlation coefficient for the whole questionnaire was 0.851 (Table 6).

\section{Discussion}

The aim of the study was to prepare a Polish adaptation of the Hill-Bone Compliance to High Blood Pressure Therapy Scale and to validate the instrument in a group of HBP patients. The Hill-Bone Compliance to High
Blood Pressure Therapy Scale showed sufficient psychometric quality in different aspects of acceptability, reliability and validity. The validation was based on determining a routine psychometric characteristic Cronbach's alpha, measuring the instrument's internal consistency. The internal consistency of the Polish adaptation of the Hill-Bone Compliance to High Blood Pressure Therapy Scale was tested at 0.851. This value is similar to the original instrument's internal consistency and to values obtained in validation studies of other language versions and cultural adaptations performed in Turkey (0.72) [15, 16], South Africa (0.77) [17] and Germany (0.73) [18]. However, in the adaptation by Krauser-Wood et al. it was found that the scale has sufficient internal consistency and factorial construct validity only for the medication-taking subscale [19]. Therefore, the authors recommend studying adherence using only this subscale (Hill-Bone Scale Short Form - SF). In the Polish adaptation, the Hill-Bone Scale was shown to have sufficient construct and criterion validity, which means HBSC scores were predictive of BP outcomes and were significantly correlated with other theoretically selected variables. The HBP scale also demonstrated high internal consistency when used in South Africa or when 
Table 4 Factor loadings for each question in the questionnaire

\begin{tabular}{llll}
\hline Question & Factor 1 & Factor 2 & Factor 3 \\
\hline Question_2 & $\mathbf{0 . 3 5}$ & -0.04 & 0.20 \\
Question_5 & $\mathbf{0 . 5 1}$ & 0.22 & 0.41 \\
Question_8 & $\mathbf{0 . 5 9}$ & 0.29 & 0.07 \\
Question_9 & $\mathbf{0 . 8 3}$ & 0.08 & 0.05 \\
Question_11 & $\mathbf{0 . 8 8}$ & 0.16 & -0.04 \\
Question_12 & $\mathbf{0 . 6 7}$ & 0.18 & 0.09 \\
Question_13 & $\mathbf{0 . 4 7}$ & 0.01 & 0.09 \\
Question_1 & 0.11 & $\mathbf{0 . 8 0}$ & 0.02 \\
Question_6 & -0.22 & $\mathbf{0 . 4 4}$ & 0.38 \\
Question_7 & 0.54 & $\mathbf{0 . 6 5}$ & -0.07 \\
Question_10 & 0.31 & $\mathbf{0 . 7 2}$ & 0.30 \\
Question_14 & 0.05 & $\mathbf{0 . 7 6}$ & -0.09 \\
Question_3 & 0.11 & -0.15 & $\mathbf{0 . 7 1}$ \\
Question_4 & -0.05 & 0.17 & $\mathbf{0 . 7 4}$ \\
Expl.Var & 3.33 & 2.61 & 1.55 \\
Prp.Totl & 0.24 & 0.19 & 0.11 \\
\hline
\end{tabular}

1-How often do you forget to take your HBP medicine? 2-How often do you decide NOT to take your HBP medicine? 3-How often do you eat salty food? 4-How often do you add salt to your food before you eat it? 5 -How often do you eat fast food? 6-How often do you make the next appointment before you leave the doctor's office? 7-How often do you miss scheduled appointments? 8-How often do you forget to get prescriptions filled? 9-How often do you run out of HBP pills? 10-How often do you skip your HBP medicine before you go to the doctor? 11-How often do you miss taking your HBP pills when you feel better? 12-How often do you miss taking your HBP pills when you feel sick? 13-How often do you take someone else's HBP pills? 14-How often do you miss taking your HBP pills when you are careless?

translated into Turkish [16, 17]. As to the interpretation of the results obtained, it should be emphasized that the psychometric qualities of the Polish adaptation of the Hill-Bone Compliance to High Blood Pressure Therapy Scale are superior to those obtained in other language versions and cultural adaptations. For example, the German validation of the HBP scale showed floor effects and poor ability to predict medication adherence for nearly every third participant [18]. According to some authors, the optimum internal consistency is indicated by Cronbach's alpha at $\geq 0.90$; values $\geq 0.80$ are considered good, $\geq 0.70$ - acceptable, $\geq 0.60$ - questionable, $\geq 0.50$ - poor and $<0.50$ - unacceptable [22]. Therefore, the Polish adaptation of the Hill-Bone Compliance to High

Table 5 Spearman's rank correlation coefficients for the scale total and the medication taking subscale score on the one hand, and systolic and diastolic blood pressure on the other

\begin{tabular}{llllll}
\hline Variable & \multicolumn{2}{l}{ Systolic blood pressure } & & \multicolumn{2}{l}{ Diastolic blood pressure } \\
\cline { 2 - 3 } \cline { 5 - 6 } & $\mathrm{R}$ & $\mathrm{p}$-value & & $\mathrm{R}$ & $\mathrm{p}$-value \\
\hline Medicine taking & -0.039 & 0.679 & & -0.015 & 0.873 \\
Total score & 0.017 & 0.859 & & 0.049 & 0.599 \\
\hline
\end{tabular}

$\mathrm{R}$ - Spearman's rank correlation coefficient; $\mathrm{p}$-value - statistical significance coefficient
Table 6 Internal consistency and intraclass correlation coefficients for each question

\begin{tabular}{llll}
\hline Question & $\begin{array}{l}\text { Correlation with } \\
\text { the total score }\end{array}$ & $\begin{array}{l}\text { Cronbach's alpha } \\
\text { with the question } \\
\text { excluded }\end{array}$ & $\begin{array}{l}\text { Intraclass } \\
\text { correlation } \\
\text { coefficient }\end{array}$ \\
\hline Question 1 & 0.471 & 0.768 & 0.842 \\
Question 2 & 0.209 & 0.788 & 0.852 \\
Question 3 & 0.140 & 0.791 & 0.820 \\
Question 4 & 0.230 & 0.784 & 0.886 \\
Question 5 & 0.541 & 0.758 & 0.830 \\
Question 6 & 0.157 & 0.808 & 0.826 \\
Question 7 & 0.599 & 0.756 & 0.787 \\
Question 8 & 0.505 & 0.765 & 0.807 \\
Question 9 & 0.543 & 0.761 & 0.855 \\
Question 10 & 0.653 & 0.746 & 0.840 \\
Question 11 & 0.582 & 0.760 & 0.888 \\
Question 12 & 0.504 & 0.763 & 0.861 \\
Question 13 & 0.320 & 0.780 & 0.807 \\
Question 14 & 0.380 & 0.774 & 0.806
\end{tabular}

1-How often do you forget to take your HBP medicine? 2-How often do you decide NOT to take your HBP medicine? 3-How often do you eat salty food? 4-How often do you add salt to your food before you eat it? 5-How often do you eat fast food? 6-How often do you make the next appointment before you leave the doctor's office? 7-How often do you miss scheduled appointments? 8-How often do you forget to get prescriptions filled? 9-How often do you run out of HBP pills? 10-How often do you skip your HBP medicine before you go to the doctor? 11-How often do you miss taking your HBP pills when you feel better? 12-How often do you miss taking your HBP pills when you feel sick? 13-How often do you take someone else's HBP pills? 14-How often do you miss taking your HBP pills when you are careless?

Blood Pressure Therapy Scale questionnaire can be considered an instrument with good psychometric qualities. A reliable and valid adherence measurement based on the patient's self-report may be helpful in daily practice.

\section{Conclusions}

The Hill-Bone Compliance to High Blood Pressure Therapy Scale proved to be suitable for use in the Polish population. The use of this screening tool for the assessment of adherence to BP treatment is recommended.

\section{Ethics approval and consent to participate}

The study protocol was approved by the Independent Bioethics Committee of the Wroclaw Medical University (decision no. KB 136/2015). All participants gave written informed consent after thorough explanation of the procedures involved.

\section{Consent for publication}

Not applicable

\section{Availability of data and materials}

The datasets supporting the conclusions of this article are included within the article. 


\section{Abbreviations}

BP: blood pressure; HBP: high blood pressure; ESC: European Society of Cardiology; SD: standard deviation; IQR: interquartile ranges; WHO: World Health Organization; HBSC scale: Hill-Bone Compliance to High Blood Pressure Therapy Scale; SF: Hill-Bone scale short form.

\section{Competing interests}

The authors declare that they have no competing interests.

\section{Authors' contributions}

IU participated in the sequence alignment and drafted the manuscript. BJP participated in the study design and coordination and helped to draft the manuscript. AC participated in the sequence alignment and helped to draft the manuscript. ASC participated in the sequence alignment. GM conceived of the study, and participated in its design and coordination. All authors read and approved the final manuscript.

\section{Acknowledgements}

The authors thank Dr Mariusz Chabowski for his assistance in preparing the paper and for his help with writing the article.

\section{Funding}

None

\section{Author details}

'Department of Clinical Nursing, Faculty of Health Science, Wroclaw Medical University, 5 Bartla Street, 51-618 Wroclaw, Poland. 'Department of Internal Medicine, Occupational Diseases and Hypertension, Wroclaw Medical University, 213 Borowska Street, 50-556 Wroclaw, Poland.

Received: 19 January 2016 Accepted: 29 April 2016

Published online: 10 May 2016

\section{References}

1. Global status report on noncommunicable diseases 2010. World Health Organization 2010. Internet: http://www.who.int/nmh/publications/ncd_ report full en.pdf. Accessed 14 Dec 2013

2. Gajewska D, Zdzieborska M, Harton A, Myszkowska-Ryciak J. The assessment of knowledge and compliance with dietary recommendations in patients with essential hypertension. Probl Hig Epidemiol. 2013;94(2):258-61.

3. Gorczyca-Michta I, Wozakowska-Kaplon B. Hypertension treatment in elderly patients. Folia Cardiologica Excerpta. 2009;4(5):279-84.

4. Berry SD, Quach L, Procter-Gray E, Kiel DP, Li W, Samelson EJ, Lipsitz LA, Kelsey JL. Poor adherence to medications may be associated with falls. J Gerontol A Biol Sci Med Sci. 2010;65A(5):553-8.

5. Tan XI, Patel I, Chang J. Review of the four item Morisky medication Adherence Scale (MMAS-4) and eight item Morisky medication Adherence Scale (MMAS-8). Innov Pharm. 2014:5(3):1-8.

6. Waeber B, Burnier M, Brunner HR. Compliance with antihypertensive therapy. Clin Exp Hypertens. 1999:21(5-6):973-85.

7. Jasinska M, Kurczewska U, Orszulak-Michalak D. Medication non-adherence in pharmaceutical care process. Opieka Farmaceutyczna. 2009:65(11):765-71.

8. Mancia G, Fagard R, Narkiewicz K, et al. 2013 ESH/ESC Guidelines for the management of arterial hypertension: the Task Force for the management of arterial hypertension of the European Society of Hypertension (ESH) and of the European Society of Cardiology (ESC). J Hypertens. 2013;31:1281-357.

9. Niiranen TJ, Hanninen MR, Johansson J, Reunanen A, Jula AM. Home-measured blood pressure is a stronger predictor of cardiovascular risk than office blood pressure: the Finn-Home study. Hypertension. 2010;55(6):1346-51.

10. Birtwhistle RV, Godwin MS, Delva MD, Casson RI, Lam M, MacDonald SE, Seguin R, Ruhland L. Randomised equivalence trial comparing three month and six month follow up of patients with hypertension by family practitioners. BMJ. 2004;328(7433):204

11. Clark CE, Smith LF, Taylor RS, Campbell JL. Nurse led interventions to improve control of blood pressure in people with hypertension: systematic review and meta-analysis. BMJ. 2010;341:c3995.

12. Reiner Z, Sonicki Z, Tedeschi-Reiner E. Physicians' perception, knowledge and awareness of cardiovascular risk factors and adherence to prevention guidelines: the PERCRO-DOC survey. Atherosclerosis. 2010;213(2):598-603.

13. Fürthauer J, Flamm M, Sönnichsen $A$. Patient and physician related factors of adherence to evidence based guidelines in diabetes mellitus type 2 , cardiovascular disease and prevention: a cross sectional study. BMC Fam Pract. 2013:14:47.

14. Culig J, Leppée M. From Morisky to Hill-bone; self-reports scales for measuring adherence to medication. Coll Antropol. 2014;38(1):55-62.

15. Kim MT, Hill MN, Bone LR, Levine DM. Development and testing of the HillBone compliance to high blood pressure therapy scale. Prog Cardiovasc Nurs. 2000:15(3):90-6.

16. Karademir M, Koseoglu $H$, Vatansever K, Van Den Akker M. Validity and reliability of the Turkish version of the Hill-Bone compliance to high blood pressure therapy scale for use in primary health care settings. Eur J Gen Pract. 2009:15(4):207-11.

17. Lambert EV, Steyn K, Stender S, Everage N, Fourie JM, Hill M. Cross-cultural validation of the Hill-Bone compliance to high blood pressure therapy scale in a South African, Primary Health Care Setting. Ethn Dis. 2006;16(1):286-91.

18. Koschack J, Marx G, Schnakenberg J, Kochen MM, Himmel W. Comparison of two self-rating instruments for medication adherence assessment in hypertension insufficient psychometric properties. J Clin Epidemiol. 2010;63(3):299-306.

19. Krousel-Wood M, Muntner P, Jannu A, Desalvo K, Re RN. Reliability of a medication adherence measure in outpatients setting. Am J Med Sci. 2005;330(3):128-33.

20. Brislin RW. Back-Translation for Cross-Cultural Research. J Cross-Cult Psychol. 1970;1(3):185-216

21. Hair JF, Anderson RE, Tatham RL, Black WC. Factorial analysis. In: Multivariate Data Analysis. Fifth edition. New Jersey: Prentice Hall; 1998.

22. Beaton DE, Bombardier C, Guillemin F, Ferraz MB. Guidelines for the process of cross-cultural adaptation of self-report measures. Spine. 2000;25(24):3186-91.

\section{Submit your next manuscript to BioMed Central and we will help you at every step:}

- We accept pre-submission inquiries

- Our selector tool helps you to find the most relevant journal

- We provide round the clock customer support

- Convenient online submission

- Thorough peer review

- Inclusion in PubMed and all major indexing services

- Maximum visibility for your research

Submit your manuscript at www.biomedcentral.com/submit
Biomed Central 